\title{
Generalized Wake Interaction Models and Optimal Control in Wind Farms
}

\author{
Lin Pan ${ }^{1,4}$, Holger Voos ${ }^{1}$, Yongping Pan ${ }^{2}$, Mohamed Darouach ${ }^{3}$ \\ 1. Interdisciplinary Centre for Security, Reliability and Trust, University of Luxembourg, Luxembourg \\ E-mail: lin.pan@uni.lu, holger.voos@uni.lu \\ 2. School of Biomedical Engineering, National University of Singapore, Singapore 117575, Singapore \\ E-mail: biepany@nus.edu.sg \\ 3. Research Center for Automatic Control of Nancy, University of Lorraine, France \\ E-mail: mohamed.darouach@univ-lorraine.fr \\ 4. School of Electric and Electronic Engineering, Wuhan Polytechnic University, Wuhan 430023, China
}

\begin{abstract}
This paper focuses on Wind Farms (WFs) optimization and Wind Energy (WE) systems of Luxembourg. The overview of wind resources in all WFs is described and a class of Improved Generalized Multiple Wake Model (IGMWM) for Coordination and Optimization Control (CnOC) of WFs are presented. Based on Wind Power (WP) assessment of WFs, the statistical methods are used to model the distribution of Wind Speed (WS) and Wind Direction (WD). Finally, some simulation figures about Wind Rose (WR) and WF optimization demonstrate the WP in detail. These assessments are expected to enhance the exploitation and utilization of WP in WFs of Luxembourg.
\end{abstract}

Key Words: Wind Energy (WE), Coordination and Optimization Control (CnOC), Wind Farms (WFs), Wind Turbine (WT), Wind Rose (WR), Wind Power (WP), Tip Speed Ratio (TSR)

\section{Introduction}

With the increase of global wind-generation capacity in the last ten years, WE has become the fastest-growing electrical energy in the world. It is well known that WE is widely recognized to be one of the most cost-efficient renewable sources of energy. In the processes of Wind Energy (WE) utilization, Wind Power (WP) assessment is a critical step. In order to enhance the effective utilization of WE, the best way is to utilize existing WFs through improving control techniques and algorithms $[1,2,3,4,5]$.

Nowadays, many researches and developments of WE harvesting systems are focused on optimizing different aspects of WTs in order to improve its Cost of Energy $(\mathrm{CoE})$, such as optimal placements of WTs in a specific area. The WTs location optimization is an effective way to reduce the wake effect of WTs and to increase the total WP of the WF. Turbines in WFs can be located along a single line, in multiple lines, in grids, in clusters or in configurations based on geographical features, prevailing WD, access requirements, environmental effects, safety, prior and future land use including ranch-land and farmland, and visual impact $[6,7]$. Therefore, some challenging problems, e.g. the electric energy product increment and the cost reduction emerged from rapid growth of the WE industry. Increasing performance of the control system by optimizing WTs controller is one of the most important ways to enhance $\mathrm{CoE}$ of WTs [6].

Generally, WTs not only generate power but also induce wakes behind their swept areas in the WFs. For the down-

This work is supported by AFR and FNR programs of Luxembourg. stream WTs, the wakes will weaken the WP generation performance. Due to the wake effect, the layout of a WFs greatly reduces the upper boundary of their potential W$\mathrm{P}$ generation. This study aims to design and optimal the WFs layout and even avoid the wake loss through the distribution of WTs. Meanwhile, because of the aerodynamic interactions among the array WTs, using control algorithms, optimizing WP capture of WFs with coordination and optimization control will no doubt increase the effective utilization of WE. One method for dealing with these aerodynamic interactions is to develop and use wake models in the distributed and optimization control algorithms. An alternative method is to develop an online control approach where each WT adjusts its own induction model coefficients according to the information of local WFs [8]. Recent years, WE is developing rapidly in Luxembourg. In view of Luxembourg locating in the western central area of European, and there are abundant wind resources to tap into Luxembourg. There are currently more than 16 WFs established in the different places of Luxembourg. As an example, Fig. 1 shows WF Wand park Gemeng Hengischt S.A., which is one of the largest WFs in Luxembourg [9]. Inspired by the above works and discussions, we argue that the key and core research contents are to operate WFs with the efficient methods, to improve WP, power quality, and energy capture of the overall WTs. Moreover, reducing complex structural electricity loading, advanced and hybrid control measures have to be applied on the WFs. All factors mentioned above motivate our research in this field.

Due to the complex interactions among the WTs, the challenging problem of WFs is the fact that the pure control on 


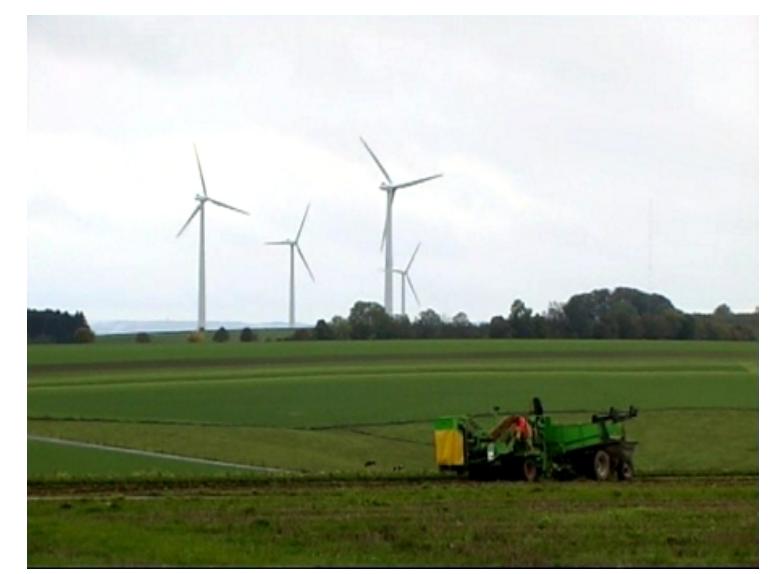

Figure 1: The portraits of WF Wand park Gemeng Hengischt S.A. in Luxembourg [9].

the single-WT is not completely sufficient. Firstly, all WTs in a farm are connected to the same Electrical Grid (EG) and each individual WT therefore has an influence on the quality or output of the generated electrical power. Secondly, there are also aerodynamic interdependencies among the WTs because each WT influences the airstream that flows through the WF. In addition, also these flows influence the wind conditions and hereby the power generation as well as the electricity structural load on other WTs. Furthermore, aerodynamic and electrical interaction among WTs in a WF also result in the losses of energy-capture, which have to be recovered by a suitable control measures. Therefore, the overall control strategy of WFs is currently a very important research topic and also the main objective which goes far beyond the control method of a single WT.

The main contributions of this paper are as follows. Firstly, an Improved Generalized Multiple Wake Model (IGMWM) for WFs has been put forward. Three kinds of shadowing area, i.e. No Shadowing Area (NSA), Partial Shadowing Area (PSA) and Completed Shadowing Area (CSA) have been calculated and presented with mathematic forms in detail. Further, wind power generation system is studied, and wind rose and wind weibull probability density distribution are elaborated and discussed that all focused on WFs of Luxembourg. Finally, the problem of WTs location optimization is discussed. Some simulation figures are provided to prove their effectiveness and correctness in the paper.

The paper is organized as follows: In section 2, An IGMWM is described and deduced, and wind power generation system is studied in section 3. The wind rose and wind Weibull probability density distribution are presented in section 4. Further, WFs optimization in luxembourg is discussed in 5. Finally, the conclusion is summarized in section 6 .

\section{An Improved Generalized Multiple Wake Model (IGMWM) for WFs}

In this section, we will discuss an Improved Generalized Multiple Wake Model (IGMWM) for WFs. An IGMWM wake effect is shown in Fig. 2. Fig. 2 points out the di- rection of free wind and its undisturbed wind speeds $v_{0}$. In the optimization of WFs, $W T_{i}$ is in front of other WTs. The wind speed at WTs, e.g. $W T_{j}, W T_{k}$ are affected not only by the upstream $W T_{i}$, but also by other upstream WTs.

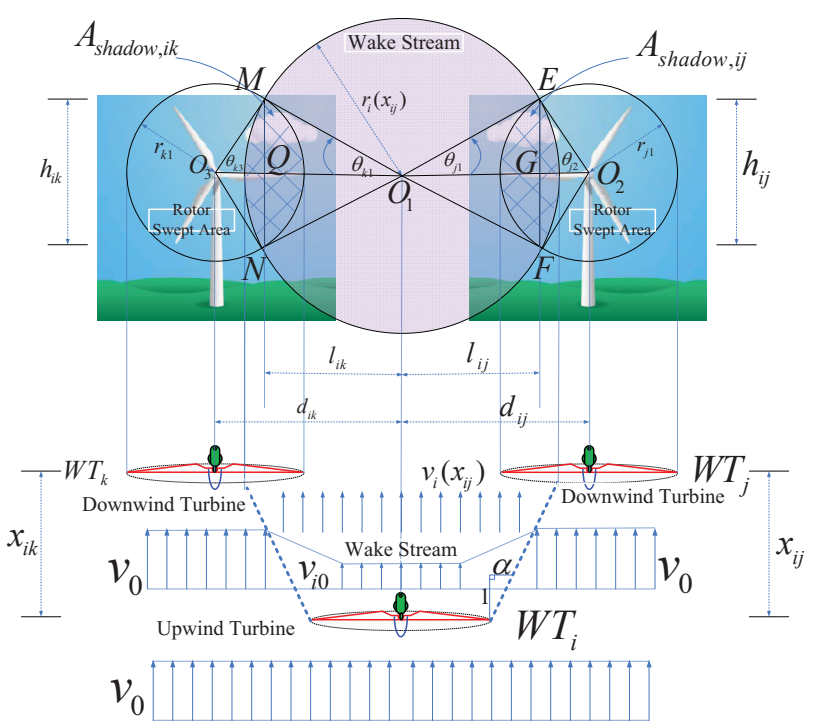

Figure 2: The portrait of Improved Generalized Multiple Wake Model (IGMWM).

This IGMWM considers the shadowed areas of the upstream WTs. This shadowing areas $\left(A_{\text {shadow }, i j}\right.$ and $\left.A_{\text {shadow, } i k}\right)$ are measures of the overlaps between the area of the circular section of the wakes shadow and the turbine area that suffers the action of this wake, an example of which is depicted in Fig. 2.

There are three kinds of shadowing area, i.e. Partial Shadowing Area (PSA), No Shadowing Area (NSA) and Completed Shadowing Area (CSA). For the situation with PSA of WTs, the input wind speed is the free wind speed in the environment mixed with the wake stream speed. For the situation with NSA of WTs, the input wind speed is the free wind speed in the environment. For the situation with CSA of WTs, the input wind speed is equal to the wake stream speed that shadows it.

If the WTs rotors have the different radiuses $\left(r_{j 1}\right.$ and $\left.r_{k 1}\right)$, then the area of the WTs being shadowed can be calculated by using the basic trigonometric relationships, which leads to the following results.

1). When $r_{i}\left(x_{i j}\right)-r_{j 1} \leq d_{i j} \leq r_{i}\left(x_{i j}\right)+r_{j 1}$, and $r_{i}\left(x_{i j}\right)-$ $r_{k 1} \leq d_{i j} \leq r_{i}\left(x_{i j}\right)+r_{k 1}, i, k=1,2, \cdots, N, n=1,2, \cdots, N$, it represents PSA. We use two different methods to solve them. One approach is:

$$
\begin{aligned}
& \sum_{i=1}^{n} \sum_{j=1}^{n} A_{\text {shadow }, i j} \\
& =\sum_{i=1}^{n} \sum_{j=1}^{n}\left[S_{\text {FanShapedRegions } O_{1} \overparen{E F} i j}\right. \\
& \left.\quad+S_{\text {FanShapedRegions } O_{2} \overparen{E F} i j}-S_{\text {Quadrilateral } O_{1} E_{2} O_{2} F i j}\right]
\end{aligned}
$$




$$
\begin{aligned}
= & \sum_{i=1}^{n} \sum_{j=1}^{n}\left[2 \frac{\theta_{j 1}}{2 \pi} \pi r_{i}^{2}\left(x_{i j}\right)+2 \frac{\theta_{j 2}}{2 \pi} \pi r_{j 1}^{2}\left(x_{i j}\right)\right. \\
& \left.-\frac{1}{2}\left|O_{1} O_{2}\right|_{i j}|E F|_{i j}\right] \\
= & \sum_{i=1}^{n} \sum_{j=1}^{n}\left[\theta_{j 1} r_{i}^{2}\left(x_{i j}\right)+\theta_{j 2} r_{j 1}^{2}\left(x_{i j}\right)-\frac{1}{2}\left|d_{i j}\right|\left|h_{i j}\right|\right] .
\end{aligned}
$$

$$
\begin{aligned}
\sum_{i=1}^{n} & \sum_{k=1}^{n} A_{\text {shadow }, i k} \\
= & \sum_{i=1}^{n} \sum_{k=1}^{n}\left[S_{\text {FanShapedRegions } O_{1} \widehat{M N} i k}\right. \\
& \left.+S_{\text {FanShapedRegions } O_{3} \widehat{M N} i k}-S_{\text {Quadrilateral }_{1} M O_{3} N i k}\right] \\
= & \sum_{i=1}^{n} \sum_{k=1}^{n}\left[2 \frac{\theta_{k 1}}{2 \pi} \pi r_{i}^{2}\left(x_{i k}\right)+2 \frac{\theta_{k 3}}{2 \pi} \pi r_{k 1}^{2}\left(x_{i k}\right)\right. \\
& \left.\quad-\frac{1}{2}\left|O_{1} O_{2}\right|_{i k}|E F|_{i k}\right] \\
= & \sum_{i=1}^{n} \sum_{k=1}^{n}\left[\theta_{k 1} r_{i}^{2}\left(x_{i k}\right)+\theta_{k 3} r_{k 1}^{2}\left(x_{i k}\right)-\frac{1}{2}\left|d_{i k}\right|\left|h_{i k}\right|\right]
\end{aligned}
$$

Herein,

$$
\theta_{j 1}=\arccos \frac{l_{i j}}{r_{i}\left(x_{i j}\right)} ; \quad \theta_{j 2}=\arccos \frac{d_{i j}-l_{i j}}{r_{j 1}} .
$$

and

$$
\left\{\begin{array}{l}
\theta_{k 1}=\arccos \frac{l_{i k}}{r_{i}\left(x_{i k}\right)}, \\
\theta_{k 3}=\arccos \frac{d_{i k}-l_{i k}}{r_{k 1}}, \\
x_{i k}=x_{i j} .
\end{array}\right.
$$

Another approach is:

$$
\begin{aligned}
& \sum_{i=1}^{n} \sum_{j=1}^{n} A_{\text {shadow }, i j} \\
& =\sum_{i=1}^{n} \sum_{j=1}^{n}\left[S_{\text {FanShapedRegions } O_{1} \overparen{E F} i j}-S_{\triangle O_{1} E F i j}\right.
\end{aligned}
$$

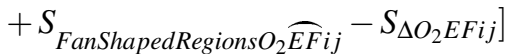

$$
\begin{aligned}
& =\sum_{i=1}^{n} \sum_{j=1}^{n}\left[2 \frac{\theta_{j 1}}{2 \pi} \pi r_{i}^{2}\left(x_{i j}\right)-\frac{1}{2}\left|O_{1} G\right|_{i j}|E G|_{i j}\right. \\
& \left.+2 \frac{\theta_{j 2}}{2 \pi} \pi r_{j 1}^{2}\left(x_{i j}\right)-\frac{1}{2}\left|O_{2} G\right|_{i j}|E G|_{i j}\right] \\
& =\sum_{i=1}^{n} \sum_{j=1}^{n}\left[\theta_{j 1} r_{i}^{2}\left(x_{i j}\right)-r_{i}^{2}\left(x_{i j}\right) \sin \theta_{j 1} \cos \theta_{j 1}\right. \\
& \left.+\theta_{j 2} r_{j 1}^{2}\left(x_{i j}\right)-r_{j 1}^{2} \sin \theta_{j 2} \cos \theta_{j 2}\right] \\
& =\sum_{i=1}^{n} \sum_{j=1}^{n}\left\{r_{i}^{2}\left(x_{i j}\right)\left[\theta_{j 1}-\frac{1}{2} \sin \left(2 \theta_{j 1}\right)\right]\right. \\
& \left.+r_{j 1}^{2}\left(x_{i j}\right)\left[\theta_{j 2}-\frac{1}{2} \sin \left(2 \theta_{j 2}\right)\right]\right\} .
\end{aligned}
$$

and

$$
\begin{aligned}
& \sum_{i=1}^{n} \sum_{k=1}^{n} A_{\text {shadow }, i k}
\end{aligned}
$$

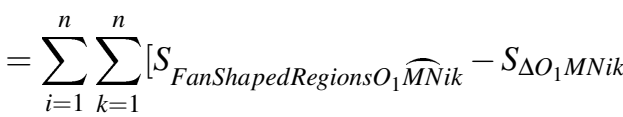

$$
\begin{aligned}
& \left.+S_{\text {FanShapedRegions } O_{3} \overparen{M N i k}}-S_{\triangle O_{3} M N i k}\right] \\
& =\sum_{i=1}^{n} \sum_{k=1}^{n}\left[2 \frac{\theta_{k 1}}{2 \pi} \pi r_{i}^{2}\left(x_{i k}\right)-\frac{1}{2}\left|O_{1} Q\right|_{i k}|M Q|_{i k}\right. \\
& \left.+2 \frac{\theta_{k 3}}{2 \pi} \pi r_{k 1}^{2}\left(x_{i k}\right)-\frac{1}{2}\left|O_{3} Q\right|_{i k}|M Q|_{i k}\right] \\
& =\sum_{i=1}^{n} \sum_{k=1}^{n}\left[\theta_{k 1} r_{i}^{2}\left(x_{i k}\right)-r_{i}^{2}\left(x_{i k}\right) \sin \theta_{k 1} \cos \theta_{k 1}\right. \\
& \left.+\theta_{k 3} r_{k 1}^{2}\left(x_{i k}\right)-r_{k 1}^{2} \sin \theta_{k 3} \cos \theta_{k 3}\right] \\
& =\sum_{i=1}^{n} \sum_{k=1}^{n}\left\{r_{i}^{2}\left(x_{i k}\right)\left[\theta_{k 1}-\frac{1}{2} \sin \left(2 \theta_{k 1}\right)\right]\right. \\
& \left.+r_{k 1}^{2}\left(x_{i k}\right)\left[\theta_{k 3}-\frac{1}{2} \sin \left(2 \theta_{k 3}\right)\right]\right\} \text {. }
\end{aligned}
$$

Herein,

$$
\left\{\begin{aligned}
\theta_{j 1} & =\arccos \left[\frac{\left|O_{1} O_{2}\right|^{2}+\left|O_{1} E\right|^{2}-\left|E O_{2}\right|^{2}}{2\left|O_{1} O_{2}\right|\left|O_{1} E\right|}\right] \\
& =\arccos \left[\frac{d_{i j}^{2}+r_{i}^{2}\left(x_{i j}\right)-r_{j 1}^{2}}{2 d_{i j} r_{i}\left(x_{i j}\right)}\right] ; \\
\theta_{j 2} & =\arccos \left[\frac{\left|O_{1} O_{2}\right|^{2}+\left|O_{2} E\right|^{2}-\left|E O_{1}\right|^{2}}{2\left|O_{1} O_{2}\right|\left|O_{2} E\right|}\right] \\
& =\arccos \left[\frac{d_{i j}^{2}+r_{j 1}^{2}-r_{i}^{2}\left(x_{i j}\right)}{2 d_{i j} r_{j 1}}\right] .
\end{aligned}\right.
$$

and

$$
\left\{\begin{aligned}
\theta_{k 1} & =\arccos \left[\frac{\left|O_{1} O_{3}\right|^{2}+\left|O_{1} M\right|^{2}-\left|M O_{3}\right|^{2}}{2\left|O_{1} O_{3}\right|\left|O_{1} M\right|}\right] \\
& =\arccos \left[\frac{d_{i k}^{2}+r_{i}^{2}\left(x_{i k}\right)-r_{k 1}^{2}}{2 d_{i k} r_{i}\left(x_{i k}\right)}\right] ; \\
\theta_{k 3} & =\arccos \left[\frac{\left|O_{1} O_{3}\right|^{2}+\left|O_{3} M\right|^{2}-\left|M O_{1}\right|^{2}}{2\left|O_{1} O_{3}\right|\left|O_{3} M\right|}\right] \\
& =\arccos \left[\frac{d_{i k}^{2}+r_{k 1}^{2}-r_{i}^{2}\left(x_{i k}\right)}{2 d_{i k} r_{k 1}}\right], \\
x_{i k} & =x_{i j} .
\end{aligned}\right.
$$

2). When $d_{i j} \geq r_{i}\left(x_{i j}\right)+r_{j 1}$, and $d_{i j} \geq r_{i}\left(x_{i j}\right)+r_{k 1}, i, k=$ $1,2, \cdots, N, n=1,2, \cdots, N$, it describes the area with NSA:

$$
\sum_{i=1}^{n} \sum_{j=1}^{n} A_{\text {shadow }, i j}=0, \text { and } A_{\text {shadow }, i k}=0,
$$

here $n=1,2, \cdots, N$.

3). When $d_{i j} \leq r_{i}\left(x_{i j}\right)-r_{j 1}$, and $d_{i j} \leq r_{i}\left(x_{i j}\right)-r_{k 1}, i, k=$ $1,2, \cdots, N, n=1,2, \cdots, N$, it represents the CSA:

$$
\sum_{i=1}^{n} \sum_{j=1}^{n} A_{\text {shadow }, i j}=\sum_{i, j=1}^{n} \pi r_{j 1}^{2} \text {, and } A_{\text {shadow }, i k}=\pi r_{k 1}^{2} \text {, }
$$


here $n=1,2, \cdots, N$.

The wind speed equation in IGMWM can be calculated using the following equation:

$$
\begin{aligned}
& \sum_{i=1}^{n} \sum_{j=1}^{n} v_{i}\left(x_{i j}\right) \\
= & \sum_{i=1}^{n} \sum_{j=1}^{n}\left[1+\left(\sqrt{1-C_{T i}}-1\right)\left(\frac{r_{j 1}}{r_{i}\left(x_{i j}\right)}\right)^{2} \frac{A_{\text {shadow }, i j}}{A_{O 1}}\right] v_{0 i}, \\
& n=1,2, \cdots, N .
\end{aligned}
$$

Meanwhile,

$$
\begin{aligned}
& \sum_{i=1}^{n} \sum_{k=1}^{n} v_{i}\left(x_{i k}\right) \\
= & \sum_{i=1}^{n} \sum_{k=1}^{n}\left[1+\left(\sqrt{1-C_{T i}}-1\right)\left(\frac{r_{k 1}}{r_{i}\left(x_{i j}\right)}\right)^{2} \frac{A_{\text {shadow }, i k}}{A_{O 3}}\right] v_{0 i}, \\
& n=1,2, \cdots, N .
\end{aligned}
$$

If the wind velocity shears are taken into consideration, the equations (15) and (16) can be improved as the following equations:

$$
\begin{aligned}
& \sum_{i=1}^{n} \sum_{j=1}^{n} v_{i}\left(x_{i j}\right) \\
& =\sum_{i=1}^{n} \sum_{j=1}^{n}\left(\frac{h_{I i}}{h_{J i}}\right)^{\alpha}[1 \\
& \left.\quad+\left(\sqrt{1-C_{T i}}-1\right)\left(\frac{r_{j 1}}{r_{i}\left(x_{i j}\right)}\right)^{2} \frac{A_{\text {shadow }, i j}}{A_{O 1}}\right] v_{0 i}, \\
& \quad n=1,2, \cdots, N .
\end{aligned}
$$

Meanwhile,

$$
\begin{aligned}
& \sum_{i=1}^{n} \sum_{k=1}^{n} v_{i}\left(x_{i k}\right) \\
& =\sum_{i=1}^{n} \sum_{k=1}^{n}\left(\frac{h_{I i}}{h_{K i}}\right)^{\alpha}[1 \\
& \left.\quad+\left(\sqrt{1-C_{T i}}-1\right)\left(\frac{r_{k 1}}{r_{i}\left(x_{i j}\right)}\right)^{2} \frac{A_{\text {shadow }, i k}}{A_{O 3}}\right] v_{0 i}, \\
& \quad n=1,2, \cdots, N .
\end{aligned}
$$

Here, $A_{O 1}$ and $A_{O 3}$ are the rotor areas of $W T_{J}$ and $W T_{K}$. $A_{\text {shadow }, i j}$ and $A_{\text {shadow, } i k}$ are the shadowing areas. The variable $h_{I i}$ and $h_{J i}$ (or $h_{K i}$ ) are the heights of the upstream WT and downstream WT, respectively.

\section{Wind Power Generation System}

Usually, the surfaces $C_{p i}-\lambda-\beta$ are used to characterize the aerodynamics of the WTs. $C_{p i}$ is the power coefficient, which is the ratio of maximum mechanical power extracted from wind at its maximum value to the total WE go through the WTs. Moreover, it is also a function including the TipSpeed Ratio (TSR) $\lambda_{i}$ and the pitch angle $\beta_{i}$. A given $C_{p i}$ can be expressed by the mechanical power $P_{m}$ and mechanical torque $T_{m}$ extracted from the wind by the $W T_{i}$ [10]:

$$
\begin{aligned}
\sum_{i=1}^{n} P_{m i} & =\sum_{i=1}^{n} \frac{1}{2} \rho A_{i} V_{w i}^{3} C_{p i}\left(\lambda_{i}, \beta_{i}\right) \\
& =\sum_{i=1}^{n} \frac{1}{2} \rho \pi R_{i}^{2} V_{w i}^{3} C_{p i}\left(\lambda_{i}, \beta_{i}\right), n=1,2, \cdots, N .
\end{aligned}
$$

The TSR $\lambda_{i}$ presents the ratio between the linear blade tip speed and the wind speed, expressed as:

$$
\sum_{i=1}^{n} \lambda_{i}=\sum_{i=1}^{n} \frac{\Omega_{t i} R_{i}}{V_{w i}}, \quad n=1,2, \cdots, N .
$$

where $\omega_{t i}$ is the turbine rotor speed, $V_{w i}$ is the wind velocity (or wind speed), $\rho$ is the density of air, $R_{i}$ is the blade length, $\Omega_{t i}$ is the turbine angular speed, and $A_{i}$ is the sweep area of the turbine blades.

$C_{p i}$ is the power coefficient, which is a nonlinear function of the TSR $\lambda_{i}$ :

$$
\begin{aligned}
& \sum_{i=1}^{n} C_{p i}\left(\lambda_{i}\right) \\
& =\sum_{i=1}^{n}\left[a_{0 i}+a_{1 i} \lambda_{i}+a_{2 i} \lambda_{i}^{2}+a_{3 i} \lambda_{i}^{3}+a_{4 i} \lambda_{i}^{4}+a_{5 i} \lambda_{i}^{5}\right] \\
& \quad n=1,2, \cdots, N .
\end{aligned}
$$

In addition, a generic equation is introduced and used the model $C_{p i}\left(\lambda_{i}\right)$ based on the modeling WT characteristics [11]:

$$
\begin{aligned}
& \sum_{i=1}^{n} C_{p i}\left(\lambda_{i}, \beta_{i}\right) \\
& =\sum_{i=1}^{n}\left[c_{1 i}\left(\frac{c_{2 i}}{\lambda_{I i}}-c_{3 i} \beta_{i}-c_{4 i}\right) \exp \left(-\frac{c_{5 i}}{\lambda_{I i}}\right)+c_{6 i} \lambda_{i}\right], \\
& \quad n=1,2, \cdots, N .
\end{aligned}
$$

here

$$
\frac{1}{\lambda_{I i}}=\frac{1}{\lambda_{i}+0.08 \beta_{i}}-\frac{0.035}{\beta_{i}^{3}+1}, \quad i=1,2, \cdots, N .
$$

and take the coefficients: $c_{1 i}=0.5176, c_{2 i}=116, c_{3 i}=0.4$, $c_{4 i}=5, c_{5 i}=21, c_{6 i}=0.0068$. For different values of the pitch angle $\beta_{i}$, the $C_{p i}-\lambda_{i}$ characteristics are illustrated in Fig 3(a). The maximum value of $C_{p i}\left(C_{\text {pmaxi }}=0.48\right)$ is got when $\beta_{i}=0^{\circ}$, and for $\lambda_{i}=\lambda_{\text {opti }}=8.1$.

The power coefficient $C_{p i}$ depends on the specific speed $\lambda_{i}$ and the angle of the blades $\beta_{i}$, and presents the aerodynamic efficiency of the WTs. The $C_{p i}$ is usually provided by the manufacturer and can use an approximate value to define. Normally, $C_{p i}$ is different from one $W T_{i}$ to another $W T_{j}$. Fig. 3(a) shows the power coefficient $C_{p i}$, which is a function of $\beta_{i}$ and $\lambda_{i}$. Fig. 3(a) indicates that existing one specific $\lambda_{\text {opti }}$ makes WTs most efficient. Different values of the pitch angle are also illustrated in this figure, e.g. $\beta_{i}=0^{\circ}, \beta_{i}=5^{\circ}, \beta_{i}=10^{\circ}, \beta_{i}=15^{\circ}, \beta_{i}=20^{\circ}$. Normally, following the $C_{\text {pmaxi }}$, the variable speed WTs reach the 


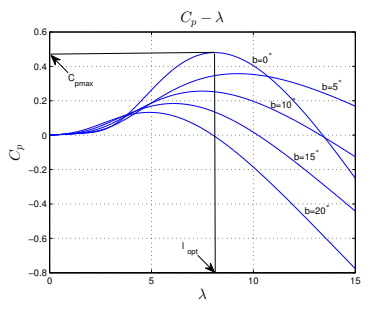

(a) Power coefficient $C_{p i}$ curve

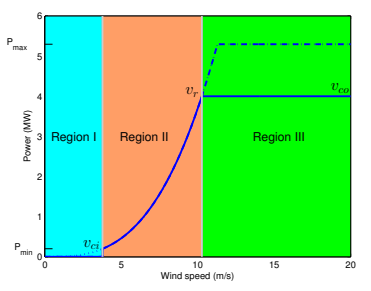

(b) Wind power curve
Figure 3: The portraits of power coefficient $C_{p i}$ curve and wind power curve in Luxembourg

rated speed and capture the maximum power by adjusting the rotor speed and the value $\lambda_{\text {opti }}$ of the system.

Then the aerodynamic torque is defined as:

$$
\begin{aligned}
& \sum_{i=1}^{n} T_{m i}=\frac{P_{m i}}{\Omega_{t i}}=\sum_{i=1}^{n} \frac{1}{2 \lambda_{i}} \rho \pi R_{i}^{3} V_{w i}^{2} C_{p i}\left(\lambda_{i}, \beta_{i}\right) \\
& =\sum_{i=1}^{n} \frac{1}{2} \rho \pi R_{i}^{3} V_{w i}^{2} C_{T i}\left(\lambda_{i}, \beta_{i}\right), \quad n=1,2, \cdots, N .
\end{aligned}
$$

where

$$
\sum_{i=1}^{n} C_{T i}\left(\lambda_{i}, \beta_{i}\right)=\sum_{i=1}^{n} \frac{C_{p i}\left(\lambda_{i}, \beta_{i}\right)}{\lambda_{i}}, \quad n=1,2, \cdots, N .
$$

Generally, the mechanical power is extracted from the wind, and their relationship between wind speed and mechanical power can be described as following:

$$
\sum_{i=1}^{n} P_{W T i}=\left\{\begin{array}{l}
0, \text { if } v_{i}<v_{c i i} \text { or } v_{i} \geq v_{c o i} ; \\
\sum_{i=1}^{n}\left[\frac{v_{i}^{3}-v_{c i i}^{3}}{v_{r i}^{3}-v_{c i i}^{3}} P_{r i}\right], \text { if } v_{c i i} \leq v_{i} \leq v_{r i} ; \\
\sum_{i=1}^{n} P_{r i}, \text { if } v_{r i} \leq v_{i} \leq v_{\text {maxi }} ; \\
0, \text { if } v_{i} \geq v_{\text {maxi }} .
\end{array}\right.
$$

where $n=1,2, \cdots, N, v_{c i}\left(\right.$ or $v_{c o i}$ ) denotes the cut-in (or cutout) wind speed $(\mathrm{m} / \mathrm{s}), v_{r i}$ is the rated wind speed $(\mathrm{m} / \mathrm{s})$, and $P_{W T r i}$ is the rated active power output of single $W T_{i}$ $(\mathrm{MW}), v_{\max i}$ is the maximum allowable cut-in speed.

Fig. 3(b) shows an ideal wind power conversion curve for WTs with $5200 \mathrm{~kW}$ in Luxembourg, which is a typical WP conversion curve of WTs, and the curve can be divided into three main stages. The first stage is Region I, the wind velocity is very small $\left(v_{i}<v_{\text {cii }}\right)$. The WT is out of operate and the WP generation is suspended because the wind velocity is below the cut-in speed, and the available WP is too low to produce the enough thrust for running the WT. The second stage is Region II, the wind velocity is bigger and bigger $\left(v_{c i i} \leq v_{i} \leq v_{r i}\right)$, the WT works at partial load below the rated power and rated speed. Therefore, Region II is also called the partial load region. The third stage is Region III, the wind velocity is stronger and stronger $\left(v_{r i} \leq v_{i} \leq v_{c o i}\right)$, the power generation reaches its rated value and remains this level. So Region III is also called the full load region. When the wind velocity is enough strong and exceeds the cut-out speed, the WT is shut off to prevent damaging the
WT structure. With the advancement of generator and WT technologies, more WTs work in the partial load regionRegion II.

The operational goal of this region is to maximize WE capture by controlling both the blade pitch angle and the TSR under varying wind speed.

\section{Wind Rose and Wind Weibull Probability Den- sity Distribution}

In this section, we describe and summarize the wind rose and wind Weibull probability density distribution focusing on WFs of Luxembourg. We get the actual and real-time data from this Website [12]. Some WE cases are reduced and the WR is simplified through merging the wind speed$s$ and wind directions in Luxembourg. These data of WE resource are collected and sorted out in the following figures. The wind rose shows the portraits of wind mean speed $(\mathrm{m} / \mathrm{s})$ in Luxembourg from Jan. 01 to Sep. 30, 2015 in Fig. 4(a) and Fig. 4(b). The most of wind mean speeds from southwest are more than $4 \mathrm{~m} / \mathrm{s}$. Whereas, a small amoun$\mathrm{t}$ of wind mean speed from northwest are approximate to $4 \mathrm{~m} / \mathrm{s}$. Therefore, wind turbines should face to southwest in WFs of Luxembourg. We continue getting the actual and real-time data from this Website [12]. The wind speed in Luxembourg are collected from Jan. 01 to Sep. 30 in 2015 and their Mean Wind Speed (MWS) is $3.6099 \mathrm{~m} / \mathrm{s}$. They are shown in the Fig. 4(b).

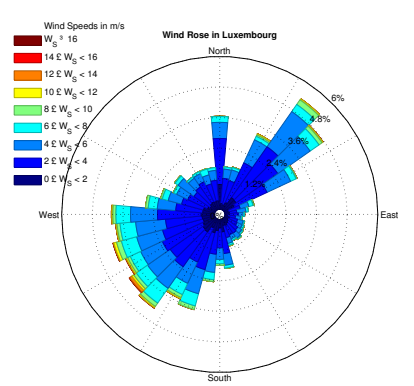

(a) $\mathrm{WD}($ North $=0$ )

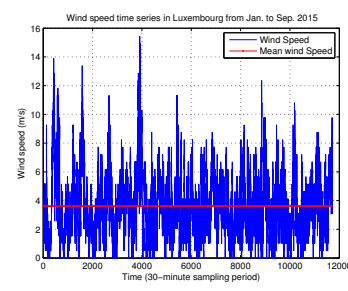

(b) Mean wind speed $(\mathrm{m} / \mathrm{s})$ Figure 4: Rose portrait of average $\mathrm{WD}($ North $=0)$ and mean wind speed $(=3.6099 \mathrm{~m} / \mathrm{s})$ in Luxembourg from Jan. 01 to Sep. 30, 2015.

The Figs. 5(a) and 5(b) show rose portraits of average WD and WD (North=0) in Luxembourg from Jan. 01 to Sep. 30, 2015. The probabilities of most of WDs from southwest are more than $3 \%$ and approximate approaches to $4 \%$. Whereas, a small amount of WD from northeast are more than 3\% and approximate approaches to $4 \%$. Therefore, WTs should face to southwest in WFs of Luxembourg.

Figs. 6(a), 6(b) and 6(c) show the parameters and fitting curve of Weibull probability distribution for the wind speed in Luxembourg. According to wind tower, measuring data and draw wind speed histograms, Using Maximum likelihood estimation method, we estimate two parameters of the Weibull distribution, i.e. $c=4.0329$, and $k=1.7616$. Then we draw the Weibull probability density distribution curve in Fig. 6(d). As seen in Fig. 6(d), the probability distribution of wind speed can be more satisfied with the Weibull distribution. 

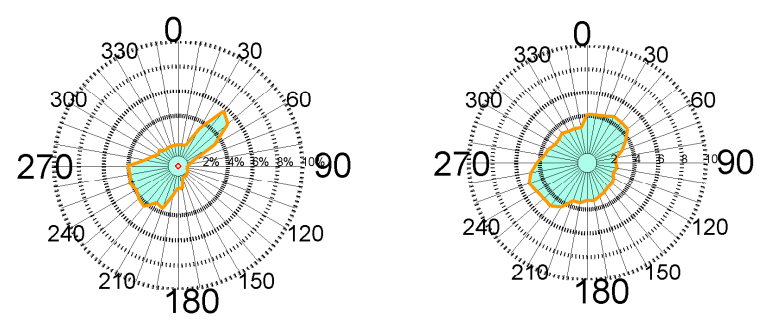

(a) Average $\mathrm{WD}($ North $=0$ )

(b) Wind Mean Speed $(\mathrm{m} / \mathrm{s})$

Figure 5: Rose portrait of average WD(North=0) and Wind Mean Speed(m/s) in Luxembourg from Jan. 01 to Sep. 30, 2015.

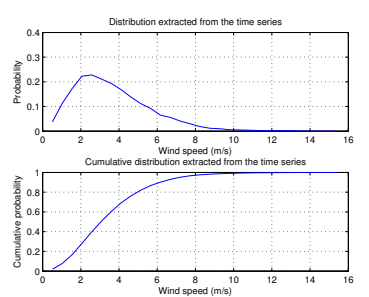

(a) Distribution and cumulative
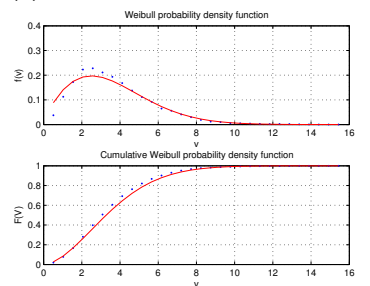

(c) Functions for Weibull density

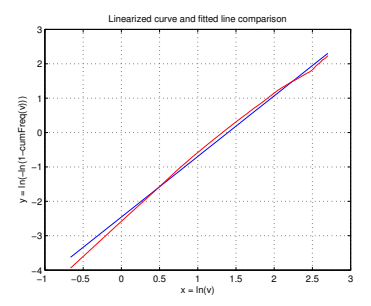

(b) Linearized curve and fitted

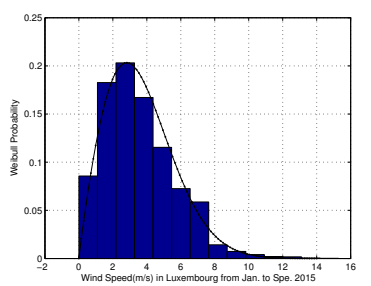

(d) Histogram and the fitted Figure 6: The portraits of Weibull probability density distribution in Luxembourg from Jan. 01 to Sep. 30, 2015.

\section{Optimal Control in Wind Farm of Luxem- bourg}

In this section, the problem of WTs location Optimal Control is discussed. When the wind flows over the WTs, there will be a region under the wake stream due to the extraction of the WE and the disturbance of the wind rotors. If one turbine is inside the wake stream of other WTs, its wind load will increase and the power output will decrease. Therefore, avoiding the wake flow, the WTs should be placed appropriately and effectively when constructing the whole WF. Here, 80 WTs are located as the similar square array in the WF. The interpretation of the dynamic effects of WF are shown in Figs. 7(a) and 7(b).

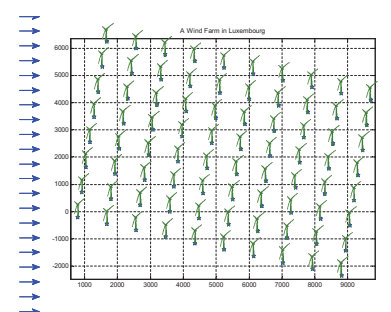

(a)

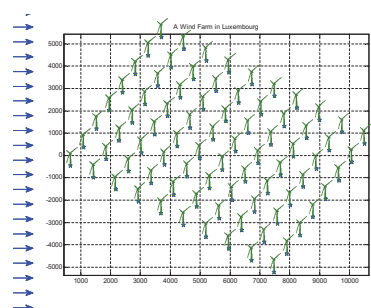

(b)
Figure 7: The portraits of Wind Farm optimization in Luxembourg

\section{Conclusions}

The paper investigates the WE systems and WFs optimization in Luxembourg. We show the overview of the wind resources in all WFs as well as present a class of IGMWM for $\mathrm{CnOC}$ of WFs. Meanwhile, the wind power generation system is studied. Moreover, the statistical method is used to model the wind speed and WD distribution for WP assessment of WFs. Further, WFs optimization in luxembourg is discussed. Some simulation figures of the wind rose and Weibull distribution demonstrate the description and assessment of WP in detail. These assessments can effectively accelerate the development of WP and utilization of WFs in Luxembourg. Next step, we will studied some new models of coordinated and optimization control for WFs. In addition, Large Eddy Simulation of WTs and WFs will also be the aim of our research.

\section{REFERENCES}

[1] S. Pookpunt and W. Ongsakul, "Optimal placement of wind turbines within wind farm using binary particle swarm optimization with time-varying acceleration coefficients," $R e$ newable Energy, vol. 55, pp. 266-276, 2013.

[2] S. Kuenzel, L. Kunjumuhammed, B. Pal, and I. Erlich, "Impact of wakes on wind farm inertial response," Sustainable Energy, IEEE Transactions on, vol. 5, no. 1, pp. 237-245, Jan 2014.

[3] R. Shakoor, M. Y. Hassan, A. Raheem, and Y.-K. Wu, "Wake effect modeling: A review of wind farm layout optimization using Jensen's model," Renewable and Sustainable Energy Reviews, vol. 58, pp. 1048-1059, 2016.

[4] L. Pan, H. Voos, Y. Li, M. Darouach, Y. Xu, and S. Hu, "A wake interaction model for the coordinated control of wind farms," in Emerging Technologies Factory Automation (ETFA), 2015 IEEE 20th Conference on, Sept 2015, pp. 1-7.

[5] L. Pan, H. Voos, Y. Li, Y. Xu, M. Darouach, and Z. Li, “A class of improved wake interaction model for the coordinated control of wind farms," in Chinese Automation Congress (CAC), 2015, Nov 2015, pp. 1322-1327.

[6] F. Ebrahimi, A. Khayatiyan, and E. Farjah, "A novel optimizing power control strategy for centralized wind farm control system," Renewable Energy, vol. 86, pp. 399-408, 2016.

[7] J. Barreiro-Gomez, C. Ocampo-Martinez, F. Bianchi, and N. Quijano, "Model-free control for wind farms using a gradient estimation-based algorithm," in Control Conference (ECC), 2015 European, July 2015, pp. 1516-1521.

[8] J. Park and K. H. Law, "A data-driven, cooperative wind far$\mathrm{m}$ control to maximize the total power production," Applied Energy, vol. 165, pp. 151-165, 2016.

[9] ENOVOS, "Enovos," http://www.enovos.eu.

[10] S. Abdeddaim and A. Betka, "Optimal tracking and robust power control of the DFIG wind turbine," International Journal of Electrical Power \& Energy Systems, vol. 49, no. 0, pp. 234-242, 2013.

[11] T. Ackermann, Wind Power in Power Systems. Wiley, 2012.

[12] I. S. U. of Science and Technology, "The Iowa Environmental Mesonet (IEM)," http://mesonet.agron.iastate.edu/. 